\title{
New diagnostic possibilities in systemic neonatal infections: metabolomics
}

\author{
Angelica Dessì ${ }^{\mathrm{a}}$, Giovanni Corsello $^{\mathrm{b}}$, Mauro Stronati $^{\mathrm{c}}$, Diego Gazzolo $^{\mathrm{d}}$, Pierluigi Caboni $^{\mathrm{e}}$, \\ Roberta Carboni ${ }^{a}$, Vassilios Fanos ${ }^{\mathrm{a}, *}$ \\ a Neonatal Intensive Care Unit, Puericulture Institute and Neonatal Section, Azienda Ospedaliera Universitaria, University of Cagliari, Cagliari, Italy \\ ${ }^{b}$ Operative Unit of Pediatrics and Neonatal Intensive Therapy, Mother and Child Department, University of Palermo, Palermo, Italy \\ ${ }^{\mathrm{C}}$ Neonatal Unit and Neonatal Intensive Care Unit, Maternal-Infant Department, Fondazione IRCCS Policlinico San Matteo, Pavia, Italy \\ ${ }^{\mathrm{d}}$ Department of Maternal, Fetal and Neonatal Health, C. Arrigo Children's Hospital, Alessandria, Italy \\ e Department of Life and Environmental Sciences, University of Cagliari, Cagliari, Italy
}

\section{A R T I C L E I N F O}

\section{Keywords:}

Metabolomics

Sepsis

Neonatal infections

Newborn

\begin{abstract}
A B S T R A C T
Systemic neonatal infection is a serious complication in preterm and term infants and is defined as a complex clinical syndrome caused by bacteria, fungi and virus. Sepsis remains among the leading causes of death in both developed and underdeveloped countries above all in the neonatal period. Earlier diagnosis may offer the ability to initiate treatment to prevent adverse outcomes. There have been many studies on various diagnostic haematological markers like acute phase reactants, C-reactive protein, procalcitonin, interleukins and presepsin. However, there is still no single test that satisfies the criteria as being the ideal marker for the early diagnosis of neonatal sepsis. In this regard, metabolomic analysis seems to be a promising method for determining metabolic variations correlated with systemic neonatal infections.
\end{abstract}

(c) 2014 Elsevier Ireland Ltd. All rights reserved.

\section{Introduction}

Sepsis is a clinical syndrome characterized by a series of immunological, metabolic, haemodynamic and respiratory alterations secondary to an infective process caused by an abnormal systemic inflammatory response syndrome (SIRS) in the organism. Systemic neonatal infections are an important long-term cause of mortality and morbidity for preterm and hospitalized newborn babies. In the United States the annual incidence of serious sepsis has been estimated at $0.3 \%$ and neonatal mortality is $10.3 \%$, with most deaths occurring within 48 hours from the onset of the infection [1]. Mortality rates vary depending on the organisms considered and the data of the National Institute of Child Health and Human Development Neonatal Research Network report mortality rates between 10 and $20 \%$ for gram positive infections, $36 \%$ for gram negative ones and $32 \%$ for fungal infections [2]. In the field of systemic neonatal infections, besides those caused by bacteria and fungi, also to be considered are those caused by viruses that can be transmitted from mother to child (CMV, HSV1, HSV2) and those caused by viruses capable of causing bronchiolitis and respiratory pathologies with viremia (such as the respiratory syncytial virus) [3]. The perinatal period is a time at risk for exposure to various virulent organisms that may come from the uterus, the birth canal, hospitalization, invasive procedures and family members and visitors. The high

\footnotetext{
* Corresponding author: Vassilios Fanos, MD, Professor of Pediatrics, Chief Neonatal Intensive Care Unit, Puericulture Institute and Neonatal Section, University of Cagliari, Cagliari, Italy. Tel.: +39 070/5109 3403.

E-mail address: vafanos@tiscali.it, vafanos@tin.it (V. Fanos).
}

incidence and seriousness of neonatal systemic infections are to be attributed to the reciprocal influence of different factors such as immaturity of the immune defence mechanisms, especially weak in preterms, and the complex interaction between the infecting pathogen and the host. Sepsis is a clinical condition caused by infectious microorganisms and induced by mediators of inflammation that cause alteration of the immune, inflammatory and coagulative equilibrium [4]. Neonatal sepsis concerns infections with onset within the first 28 days of life for a term neonate and within 4 weeks after the predicted birth date for preterms; in this period early diagnosis is complicated by the presence of non-infective conditions similar to those of sepsis, especially in low birth weight preterms and by the lack of optimal diagnostic tests. Despite the many and extensive investigations that have been performed in the last decades, there is still no single test that satisfies the criteria as being the ideal marker for the early diagnosis of neonatal sepsis. Since neonatal sepsis is a complex disorder involving different organs which leads to wide variations in the organism's metabolites, analysis of the entire metabolome is a promising method for determining metabolic variations correlated with sepsis.

\section{Systemic infections and metabolomics}

Systemic infections are among the major causes of neonatal mortality and long-term disability in very low birth weight (VLBW) children. Although biomolecular knowledge on the pathophysiology of sepsis has improved over the years, for this pathology no markers making early diagnosis possible have yet been identified nor have prognostic biomarkers of high sensitivity and specificity been 
Table 1

Metabolomic studies that have analyzed the metabolic profiles of septic patients.

\begin{tabular}{|c|c|c|c|c|}
\hline Author & Population study & Sample & Metabolomic analysis & Metabolite results \\
\hline Mickiewicz et al. 2013 [13] & $\begin{array}{l}60 \text { septic shock vs } \\
40 \text { SIRS vs } 40 \text { control } \\
\text { pediatric patients }\end{array}$ & Serum & ${ }^{1} \mathrm{H}-\mathrm{NMR}$ & $\begin{array}{l}\text { 2-hydroxybutyrate, 2-hydroxyisovalerate, lactate, } \\
\text { glucose, 2-oxoisocaproate, creatine, creatinine, histidine, } \\
\text { and phenylalanine }\end{array}$ \\
\hline Fanos et al. 2013 [12] & $\begin{array}{l}12 \mathrm{HCMV} \text { infected vs } \\
11 \text { control newborns }\end{array}$ & Urine & ${ }^{1} \mathrm{H}-\mathrm{NMR}$ & $\begin{array}{l}\text { Myoinositol, glycine, 3-hydroxybutyrate, } \\
\text { 3-aminoisobutyrate, creatine, taurine and betaine }\end{array}$ \\
\hline Schmerler et al. 2012 [11] & $\begin{array}{l}74 \text { SIRS vs } 69 \text { septic vs } \\
16 \text { control human adults }\end{array}$ & Blood & LC-GC/MS & Acylcarnitines and glycerophosphatidylcholines \\
\hline $\begin{array}{l}\text { Izquierdo-García et al. } 2011 \\
\text { [14] }\end{array}$ & $\begin{array}{l}14 \text { septic vs } 14 \text { control } \\
\text { rats }\end{array}$ & $\begin{array}{l}\text { Lung tissue, } \\
\text { bronchoalveolar lavage } \\
\text { fluid and serum }\end{array}$ & ${ }^{1} \mathrm{H}-\mathrm{NMR}$ & Alanine, creatine, phosphoethanolamine and myoinositol \\
\hline Liu et al. 2010 [10] & 40 septic vs control rats & Plasma & UPLC-Q-TOF-MS & $\begin{array}{l}\text { Hypoxanthine, indoxyl sulfate, glucuronic acid, gluconic } \\
\text { acid, proline, uracil, nitrotyrosine, uric acid and } \\
\text { trihydroxy cholanoic acid }\end{array}$ \\
\hline Lin et al. 2009 [9] & $\begin{array}{l}40 \text { septic vs } 20 \text { control } \\
\text { rats }\end{array}$ & Serum & ${ }^{1} \mathrm{H}-\mathrm{NMR}$ & $\begin{array}{l}\text { Lactate, alanine, acetate, acetoacetate, hydroxybutyrate } \\
\text { and formate }\end{array}$ \\
\hline
\end{tabular}

${ }^{1} \mathrm{H}$ NMR, ${ }^{1} \mathrm{H}$-nuclear magnetic resonance; HCMV, human cytomegalovirus; SIRS, systemic inflammatory response syndrome; UPLC-Q-TOF-MS, ultra performance liquid chromatography quadrupole time-of-flight mass spectrometry; LC, liquid chromatography; GC/MS, gas chromatography/mass spectrometry.

found [5]. In recent years, several single biochemical parameters of sepsis have been proposed such as C-reactive protein (CRP), white cell count, fibrinogen, D-dimer, platelet count, procalcitonin (PCT), serum amyloid protein, interleukins and presepsin [6,7]. However, certain recently developed investigative techniques provide a holistic vision of the biological system in its entirety. Among these, metabolomics (the most recent of the omic sciences) appears to be the most promising. It is defined as the study of the complex system of metabolites and, besides taking into consideration one or a few metabolites and their relative metabolic processes, it is capable of examining the entire metabolic profile of an individual. Recent studies show the efficacy of metabolomics in providing significant information useful in arriving at an early diagnosis of several neonatal pathologies [8]. The need for a highly sensitive and predictive test in the neonatal period for identifying and correctly classifying the highest possible number of septic neonates make metabolomics a method of great diagnostic potential. Works on metabolomics concerning sepsis conducted on animals and humans have recently been published (Table 1). It is interesting to see in the different studies that among the metabolites that characterize septic patients there are clear analogies. Firstly, both in studies on rats $[9,10]$ and human patients [11-13] increases in metabolites in septic patients compared to controls (in plasma and urine) which are part of the oxidative metabolism of fatty acids (such as hydroxybutyrate, acylcarnitines and acetoacetate) have been found. It is known that fatty acids, once released from adipose tissue and transported into the cytoplasm of hepatic and muscle cells, are activated in the form of esters of coenzyme A. The latter, by means of a complex mechanism of transesterification with carnitine, cross the mitochondrial membrane where they enter the beta oxidation cycle with the formation of acetyl-CoA. The latter is used by the liver for the synthesis of the ketone bodies (3-hydroxibutyrate and acetoacetate) while in muscle tissue it directly enters the cycle of the tricarboxylic acids with freeing of $\mathrm{CO}_{2}$ and $\mathrm{H}_{2} \mathrm{O}$ and formation of ATP. It has been observed that conditions of stress of the organism that cause an increase in lipolysis and/or protein catabolism to satisfy the need for a surplus of energy, as in the case of sepsis, determine an increase in renal excretion of carnitine and other metabolites involved in the oxidative metabolism of fatty acids. In several works [9,12-14], lactate, together with glucose and myoinositol content in the blood and urine of septic patients compared to controls has also been found to be higher. These results can be explained by taking into account that sepsis corresponds to a systemic inflammation caused by the anomalous presence of bacterial or viral antigens and involves different mechanisms, such as hypoxia and oxidative stress. At the outset of the infection, inhibition of glycogen synthesis is observed and this leads to a major availability of global glucose and greater cell absorption [15]. Glucose absorption appears increased in organs containing a large population of phagocytic cells (liver, spleen, intestine, lungs). Sepsis is also capable of significantly modifying cytoplasmatic glycolysis at the transcriptional level. In particular, the key enzymes of glycolysis and those of the mitochondrial respiratory chain are temporarily under-expressed. It has been seen that in the muscle of septic rats the activity of pyruvate dehydrogenase is reduced, at the same time with an increase in the activity of pyruvate dehydrogenase kinase (PDK), its inhibitor. The net result of all these changes is the reduction of the pyruvate that enters the mitochondria while the conversion of pyruvate into lactate is accelerated. This is confirmed by the fact that in patients with septic shock there is an increased use of glucose and an increase in the production of lactate at the anaerobic level $[10,14]$. Briefly stated, alterations in the glucose metabolism in critical conditions can be seen as a redistribution of glucose consumption from the mitochondrial oxidative phosphorylation to other metabolic pathways, such as the production of lactate and the pentose phosphate pathway. All this would explain the same metabolic pattern found in several septic patients assessed by means of metabolomics. Still few are the works published in the literature on metabolomics and systemic infections in pediatric patients and in particular in neonates. In a quite recent study by Mickiewicz et al. [13] it is demonstrated that the metabolomic approach may be useful in the diagnosis and prognosis of septic shock in pediatric intensive care units (PICUs). The authors examined serum samples collected from 60 patients with septic shock (by Gram- and/or Gram+), 40 patients with SIRS and 40 healthy children by nuclear magnetic resonance spectroscopy spectra. Some of the metabolite concentrations identified in serum samples changed markedly, thus indicating their influence on the separation between patient groups. These metabolites represent a composite biopattern of the pediatric metabolic response to septic shock and might be considered as the basis for a biomarker panel for the diagnosis of septic shock and its mortality in PICUs. In another work by Fanos et al. [12], urine samples of neonates infected by human cytomegalovirus (HCMV) were analyzed by means of ${ }^{1} \mathrm{H}$-nuclear magnetic resonance spectroscopy combined with multivariate statistical analysis. The authors suggest 
the use of metabolomics as a new and useful instrument also in the study of congenital HCMV infections. In the literature we can also find preliminary data on metabolomics and viral bronchiolitis in the neonate [16]. As concerns bacterial pneumonia infections, it is interesting to see that thanks to the use of metabolomics it has been possible to differentiate the urine metabolic profile in pneumonia caused by pneumococcus from the metabolic profiles of viral pneumonia and other bacterial forms [17].

\section{Conclusions}

Present-day methods and procedures for the diagnosis of systemic neonatal infections are hindered by low sensitivity and long response times. They are inadequate to satisfy the need to proceed to a rapid, timely and efficacious therapeutic treatment. Metabolomics is showing promise of becoming a most effective method in neonatology, especially because it not only allows early diagnosis of different pathologies and their monitoring, but is even capable of predicting their onset and the capacity to respond to therapy, thus making possible a tailored management of complex pediatric disorders. Taking into account all the studies published up to now, metabolomics appears to be a promising and useful instrument also in the diagnosis of sepsis. However, it will be necessary in the future to confirm these results by means of further studies so as to arrive at a more detailed picture from the metabolic standpoint, especially in systemic neonatal infections.

\section{Conflict of interest}

The authors declare that they do not have conflict of interest.

\section{References}

[1] Shane AL, Stoll BJ. Recent developments and current issues in the epidemiology, diagnosis, and management of bacterial and fungal neonatal sepsis. Am J Perinatol 2013;30(2):131-41.
[2] Stoll BJ, Hansen N, Fararoff AA, Wright L, Carlo WA. Late-onset sepsis in very low birth weight neonates: the experience of the NICHD neonatal research network. Pediatrics 2002;110:285-91.

[3] Tremolada S, Delbue S, Ferrante P. Viral infections of the fetus and newborn infant. Pediatr Med Chir 2008;30(4):177-91.

[4] Chirico G, Cortinovis S, Fonte C, Giudici G. Bacterial sepsis. J Chemother 2007;19(2):28-30.

[5] Shah BA, Padbury JF. Neonatal sepsis: An old problem with new insights. Virulence 2013;5(1) [Epub ahead of print].

[6] Raimondi F, Ferrara T, Maffucci R, Milite P, Del Buono D, Santoro P, et al. Neonatal sepsis: A difficult diagnostic challenge. Clin Biochem 2011;44(7): 463-4.

[7] Mussap M, Puxeddu E, Burrai P, Noto A, Cibecchini F, Testa M, et al Soluble CD14 subtype (sCD14-ST) presepsin in critically ill preterm newborns: preliminary reference ranges. J Matern Fetal Neonatal Med 2012;25(5):51-3.

[8] Mussap M, Antonucci R, Noto A, Fanos V. The role of metabolomics in neonatal and pediatric laboratory medicine. Clin Chim Acta 2013;426:127-38.

[9] Lin ZY, Xu PB, Yan SK, Meng HB, Yang GJ, Dai WX, et al. A metabonomic approach to early prognostic evaluation of experimental sepsis by (1)H NMR and pattern recognition. NMR Biomed 2009 Jul;22(6):601-8.

[10] Liu XR, Zheng XF, Ji SZ, Lv YH, Zheng DY, Xia ZF, et al. Metabolomic analysis of thermally injured and/or septic rats. Burns 2010;36(7):992-8.

[11] Schmerler D, Neugebauer S, Ludewig K, Bremer-Streck S, Brunkhorst FM, Kiehntopf M. Targeted metabolomics for discrimination of systemic inflammatory disorders in critically ill patients. J Lipid Res 2012;53(7):1369-75.

[12] Fanos V, Locci E, Noto A, Lazzarotto T, Manzoni P, Atzori L, et al. Urinary metabolomics in newborns infected by human cytomegalovirus: a preliminary investigation. Early Hum Dev 2013;89(1):S58-61.

[13] Mickiewicz B, Vogel HJ, Wong HR, Winston BW. Metabolomics as a novel approach for early diagnosis of pediatric septic shock and its mortality. Am J Respir Crit Care Med 2013;187(9):967-76.

[14] Izquierdo-García JL, Nin N, Ruíz-Cabello J, Rojas Y, de Paula M, López-Cuenca $\mathrm{S}$, et al. A metabolomic approach for diagnosis of experimental sepsis. Intensive Care Med 2011;37(12):2023-32.

[15] Wallington J, Ning J, Titheradge MA. The control of hepatic glycogen metabolism in an in vitro model of sepsis. Mol Cell Biochem 2008;308:18392.

[16] Atzei A, Atzori L, Moretti C, Barberini L, Noto A, Ottonello G, et al Metabolomics in paediatric respiratory diseases and bronchiolitis. J Matern Fetal Neonatal Med 2011;24(2):59-62.

[17] Slupsky CM, Rankin KN, Fu H, Chang D, Rowe BH, Charles PG, et al. Pneumococcal pneumonia: potential for diagnosis through a urinary metabolic profile. J Proteome Res 2009;8(12):5550-8. 